\title{
Characterization of a salt-induced DhAHP, a gene coding for alkyl hydroperoxide reductase, from the extremely halophilic yeast Debaryomyces hansenii Hsiu-fung Chao ${ }^{1}$, Yung-fu Yen ${ }^{* 2}$ and Maurice SB Ku*2
}

Address: ${ }^{1}$ Graduate Institute of Agriculture, National Chiayi University, Chiayi, Taiwan, Republic of China 60004 and ${ }^{2}$ Graduate Institute of Agricultural Biotechnology, National Chiayi University, Chiayi, Taiwan, Republic of China 60004

Email: Hsiu-fung Chao - S0931402@mail.ncyu.edu.tw; Yung-fu Yen* - yfyen@mail.ncyu.edu.tw; Maurice SB Ku* - mku@mail.ncyu.edu.tw

* Corresponding authors

Published: 28 August 2009

BMC Microbiology 2009, 9:182 doi:10.1186/147|-2180-9-182
Received: 2 March 2009

Accepted: 28 August 2009

This article is available from: http://www.biomedcentral.com//47/-2/80/9//82

(c) 2009 Chao et al; licensee BioMed Central Ltd.

This is an Open Access article distributed under the terms of the Creative Commons Attribution License (http://creativecommons.org/licenses/by/2.0), which permits unrestricted use, distribution, and reproduction in any medium, provided the original work is properly cited.

\begin{abstract}
Background: Debaryomyces hansenii is one of the most salt tolerant species of yeast and has become a model organism for the study of tolerance mechanisms against salinity. The goal of this study was to identify key upregulated genes that are involved in its adaptation to high salinity.

Results: By using forward subtractive hybridization we have cloned and sequenced DhAHP from $D$. hansenii that is significantly upregulated during salinity stress. DhAHP is orthologous to the alkly hydroperoxide reductase of the peroxiredoxin gene family, which catalyzes the reduction of peroxides at the expense of thiol compounds. The full-lengthed cDNA of DhAHP has 674 bp of nucleotide and contains a 516 bp open reading frame (ORF) encoding a deduced protein of 172 amino acid residues $(18.3 \mathrm{kDa})$. D. hansenii Ahp is a cytosolic protein that belongs to the Ahp of the I-Cys type peroxiredoxins. Phylogentically, the DhAhp and Candida albicans Ahp I I (Swiss-Prot: Q5AF44) share a common ancestry but show divergent evolution. Silence of its expression in $D$. hansenii by RNAi resulted in decreased tolerance to salt whereas overexpression of DhAHP in D. hansenii and the salt-sensitive yeasts Saccharomyces cereviasiae and Pichia methanolica conferred a higher tolerance with a reduced level of reactive oxygen species.

Conclusion: In conclusion, for the first time our study has identified alkly hydroperoxide reductase as a key protein involved in the salt tolerance of the extremely halophilic $D$. hansenii. Apparently, this enzyme plays a multi-functional role in the yeast's adaptation to salinity; it serves as a peroxidase in scavenging reactive oxygen species, as a molecular chaperone in protecting essential proteins from denaturation, and as a redox sensor in regulating $\mathrm{H}_{2} \mathrm{O}_{2}$-mediated cell defense signaling.
\end{abstract}

\section{Background}

Debaryomyces hansenii is an ascomycetous salt- and high $\mathrm{pH}$-tolerant yeast that has been defined as halotolerant or halophilic [1]. It was isolated from saline environments such as sea water [2] or concentrated brines [3], representing one of the most salt tolerant species of yeasts. This marine yeast can tolerate salinity levels up to $24 \%$ (4.11 M) of $\mathrm{NaCl}$ [2]. In contrast, growth of the Baker's yeast 
Saccharomyces cerevisiae is severely inhibited when salinity reaches $10 \% \mathrm{NaCl}$ [3]. Thus, $D$. hansenii has become a model organism for the study of salt tolerance mechanisms in eukaryotic cells [4]. It is now well recognized that the mechanisms by which all organisms achieve osmotic and ionic equilibrium are mediated by orthologous mechanisms based on conserved biochemical and/or physiological functions that are inherently necessary for essential metabolic processes [5].

Under saline conditions, D. hansenii accumulates large amounts of $\mathrm{Na}^{+}$without being intoxicated even when $\mathrm{K}^{+}$ is present at low concentration in the environment [6]. In fact, $\mathrm{Na}^{+}$improves growth and protects $D$. hansenii in the presence of additional stress factors [1]. For example, at high or low temperature and extreme $\mathrm{pH}$ growth of the yeast is improved by the presence of $1 \mathrm{M} \mathrm{NaCl}$ [7]. It has been clearly shown that sodium ions are less toxic for $D$. hansenii as compared with other organisms; therefore, it is considered a 'sodium-includer' organism [8]. The reduced toxic effect by $\mathrm{Na}^{+}$and its accumulation at high levels under high salt is probably indicative of an adaptive strategy of $D$. hansenii for growth in hypersaline environments [9]. The organism must posses an array of advantageous characteristics that collectively confer its high halotolerance. Earlier studies have identified a number of saltrelated genes in the extreme halophilic yeast $D$. hansenii, such as HOG1 (MAP kinase involved in high-osmolarity glycerol synthesis pathway) [10], ENA1 and ENA2 (plasmamembrane $\mathrm{Na}^{+}-$ATPase [11], GPD1 and GPP (NADglycerol-3-phosphate dehydrogenase and glycerol-3phosphatase) [12], NHX1 (vacuolar $\mathrm{Na}^{+}$antiporter) [13] and KHA1 $\left(\mathrm{Na}^{+} / \mathrm{H}^{+}\right.$antiporter $)[14]$. As expected, most of these salt-upregulated genes are involved in osmoregulation or transport of ions. However, the collective underlying mechanisms by which $D$. hansenii tolerates high levels of $\mathrm{NaCl}$ remain unkown.

All aerobic organisms require oxygen for efficient production of energy, but at the same time the organisms are constantly exposed to oxidative stress. This can be caused by partially reduced forms of molecular oxygen (e.g. superoxide radical, hydrogen peroxide and hydroxyl radical), known as reactive oxygen species (ROS), which are generated as normal metabolism byproducts, especially from respiration [15]. These ROS are highly reactive molecules that are capable of damaging cellular constituents such as DNA, RNA, lipids and proteins [16]. In adaptation to oxidative stress, aerobic organisms have evolved multiple enzymatic and non-enzymatic defense systems to protect their cellular constituents from ROS and to maintain their cellular redox state [17]. Accumulation of ROS is known to increase under many, if not all, stress conditions as the defensive scavenging systems become insufficient to cope with increasing levels of stress.
The enzymatic scavenging system for ROS involves a number of enzyme-catalyzed reactions in different cellular compartments. A series of peroxidases referred to as peroxiredoxins (Prxs) that are ancestral thiol-dependent selenium- and heme-free peroxidases [18] have been found from archaea, lower prokaryotes to higher eukaryotes. These peroxidases constitute a large family including bacterial AhpC proteins and eukaryotic thioredoxin peroxidases (TPxs) [19]. Prxs are abundant, well-distributed peroxidases that reduce $\mathrm{H}_{2} \mathrm{O}_{2}$, organic peroxides and peroxynitrite at the expense of thiol compounds. Thus, Prxs are considered alternative hydroperoxide scavenging enzymes, as they can reduce both organic and inorganic peroxides as well as oxidized enzymes. Based on the number of cysteine residues involved in catalysis, Prxs can be divided into three classes: typical 2-Cys Prxs, atypical 2Cys prxs and 1-Cys Prxs [20]. Prxs are ubiquitous proteins that use an active site Cys residue from one of the homodimers to reduce $\mathrm{H}_{2} \mathrm{O}_{2}$. The peroxidative cysteine sulfenic acid formed upon reaction with peroxide is reduced directly by glutathione. It is suggested that Prxs can act alternatively as peroxidases or as molecular chaperones by changing their molecular complexes. Furthermore, the oxidized cysteinly species, cysteine sulfenic acid, may play a dual role by acting as a catalytic intermediate in the peroxidase activity and as a redox sensor in regulating $\mathrm{H}_{2} \mathrm{O}_{2}$-mediated cell defense signaling.

Alkyl hydroperoxide reductase (Ahp) is the second known member of a class of disulfide oxidoreductases [21] and a member of the thiol-dependent peroxiredoxin family [20], which possesses activity against $\mathrm{H}_{2} \mathrm{O}_{2}$, organic peroxides, and peroxynitrite [22]. Therefore, expression of Ahp genes plays an important role in peroxide resistance (oxidative stress) in Bacillus subtilis [23], Clostridium pasteurianum [24] and Burkholderia cenocepacia [25]. Moreover, the compensatory expression of $A h p C$ in Burkholderia pseduomallei kat $\mathrm{G}$ is essential for its resistance to reactive nitrogen intermediates [26].

In this article, we report the isolation of DhAHP from the extreme halophilic yeast $D$. hansenii via subtractive hybridization of cDNA isolated from high salt treated vs. non-treated cells. Further characterization of DhAHP showed that it is a gene orthologous to alkyl hydroperoxide reductase of Candida albicans (Gene ID: 3637850 AHP11). On the basis of the deduced amino acid sequence, we propose that DhAhp be classified as an alkyl hydroperoxide reductase. Silencing of its expression in $D$. hansenii by RNAi resulted in decreased tolerance while overexpression conferred enhanced tolerance to salinity. Furthermore, overexpression of DhAHP in the salt-sensitive $S$. cerevisiae and $P$. methanolica also endowed upon their cells greater tolerance to $\mathrm{NaCl}$. These overexpression transformants exhibited reduced levels of ROS under 
salinity stress. These results suggest that the cytosolic Ahp, induced and accumulated under saline conditions, may play a key role in this extremely halophilic yeast in adaption to high salinity by scavenging ROS, serving as chaperone and mediating $\mathrm{H}_{2} \mathrm{O}_{2}$-mediated defense signaling.

\section{Results}

Characterization of salt-induced gene in $D$. hansenii In this study, forward subtractive hybridization PCR was employed to investigate the genes of $D$. hansenii that are induced by salt. The subtracted cDNA library was enriched in differentially expressed sequences after treatment with $2.5 \mathrm{M} \mathrm{NaCl}$ for $24 \mathrm{~min}$, relative to control cDNA. One of the selected clones that showed a significant increase in expression after salt induction is a homolog to the gene encoding for alkyl hydroperoxide reductase in C. albicans (Gene ID: 3637850 AHP11). This D. hansenii gene, $D h A H P$, was further characterized for its genomic organization, expression pattern and function.

\section{Cloning of full-lengthed cDNA of DhAHP}

To obtain a full-lengthed cDNA for DhAHP a forward gene specific primer (GSP) was designed and used for amplification of the 3' end of DhAHP, based on the partial sequence of the clone isolated from the subtracted cDNA library. A single DNA fragment of about 433 bp (Fig. 1A) was amplified using the primers of GeneRace 3' and forward GSP. According to the 3'-end fragment sequence, a specific reverse GSP was designed to amplify the 5 '-end of DhAHP and a fragment of 557 bp was obtained (Fig. 1B). Alignment of the 3' and 5' RACE products showed that the full-lengthed cDNA of DhAHP has 240 bp overlapped, while 59 bp of the 5 ' untranslated region (UTR) is found upstream of the first ATG codon and 99 bp of the 3' UTR

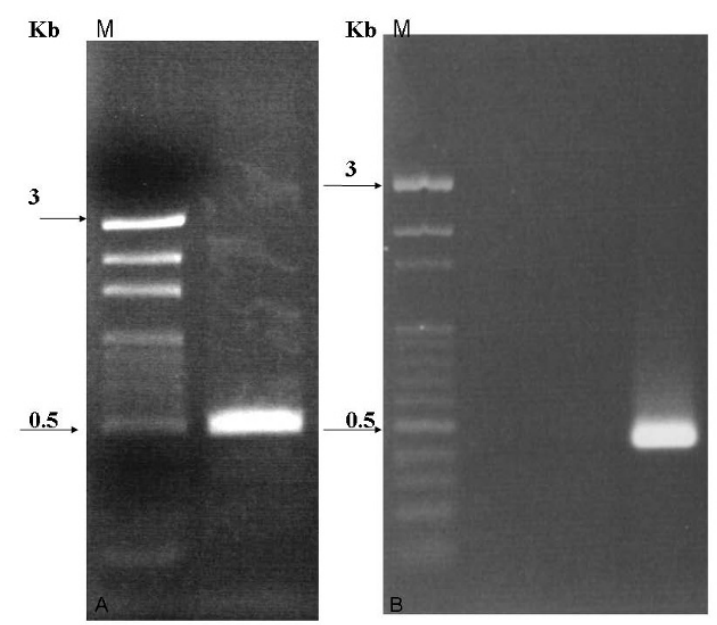

\section{Figure I}

Gel analysis of the DhAHP 3 '-end (A) and 5 '-end (B) amplification products from $D$. hansenii. is found downstream from the stop codon in the amplified sequence.

The full-lengthed cDNA of DhAHP has 674 bp of nucleotide and contains a 516 bp open reading frame (ORF) encoding a deduced protein of 172 amino acid residues (Fig. 2). The protein has an isoelectric point (pI) of 4.84 and a calculated molecular mass of $18.3 \mathrm{kDa}$. The richest amino acids are Ala (11.7\% by frequency), followed by Gly (9.4\%), Thr (8.8\%), Asp (7.6\%), Lys (7.0\%), Leu $(7.0 \%)$, Val (6.4\%) and Ile (6.4\%). Hydrophobic and hydrophilic amino acids account for $57.8 \%$ and $42.2 \%$ of the total amino acids, respectively.

\section{Characteristics of DhAHP and related genes}

The deduced $D$. hansenii Ahp amino acid sequence was compared with those of related proteins from the EMBL database using the EMBOSS alignment program. The analysis showed that the protein has $72.7 \%$ similarity to C. albicans alkyl hydroperoxide reductase (Gene ID: 3637850 AHP11). Thus, the isolated gene is homologous to the Ahp gene of $C$. albicans and is therefore named DhAHP. The DhAhp sequence was also compared with a number of previously identified Ahp and peroxiredoxin homologs from different organisms using the protein sequence alignment program CLUSTAL W. Multiple sequence alignment analysis showed that DhAhp has 58\% similarity to AHP11 (Swiss-Prot: Q5AF44) of C. albicans, $37 \%$ to peroxiredoxin of Pisum sativum (Swiss-Prot: B3GV28), 34\% to peroxiredoxin of $P$. tremula (Swiss-Prot: Q8S3L0), 33\% to PMP20 of Schizosaccharomyces pombe (Swiss-Prot: O14313), 30\% to AHP1 of S. cerevisiae (Swiss-Prot: P38013), and 25\% to Homo sapiens peroxiredoxin 5 (Swiss-Prot: P30044) (Fig. 3A). Furthermore, Cys54 , which is conserved in all related Prxs, is identified as the peroxidative cysteine in DhAhp.

Phylogenetic analysis revealed that the DhAhp protein is more homologous to yeast Ahps than to other Ahps from plants or peroxiredoxins from mammals. The DhAhp is located in the same subgroup as Ahps from yeasts, such as C. albicans and S. cerevisiae. Taken together, these results suggest that the Ahp of $D$. hansenii is more closely related to those of yeasts than to the plant Ahps or mammalian peroxiredoxins. It is conceivable that its function or enzymatic characteristics may be close to those of yeast Ahps (Fig. 3B).

\section{Genome organization and expression of DhAHP}

Southern blot analysis showed a single DNA fragment with homology to DhAHP (Fig. 4A) suggesting that it exists as a single copy in the genome of $D$. hansenii. Northern blot analysis revealed that expression of DhAHP is modulated by salt. A single transcript was detected in the cells treated with $2.5 \mathrm{M} \mathrm{NaCl}$ for $16 \mathrm{~min}$ (Fig. 4B). In con- 
ATG TCA CAA GCA CCA GTT GCA ACC CTT AAA TAC GTT CCA ATT ACT AAT GGC AAT

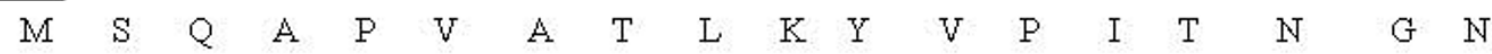
CAT GAA TTG ACC TCT TGT GCT CCT CCA CAA CCA TTA GAC TTG GCC AAA GAG TTT

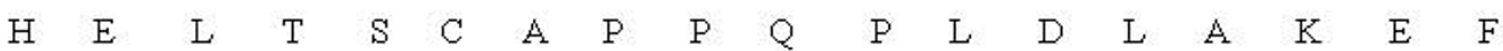
GCT GGT AAA AAA GTG CTT TTA ACT GGA GCC CCA GGA GCA TTT ACT CCT ACT TGT

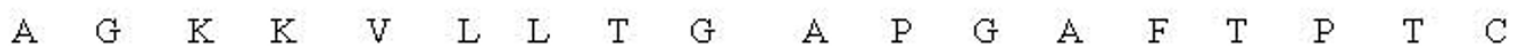
ACT GAG CAA CAT ATT CCC GAT TAT TTG GCC CAT ATT GCC GAT TTC AAG GCC AAG

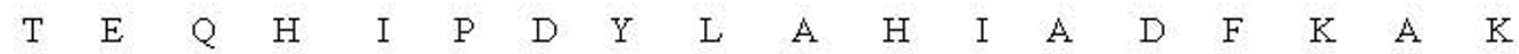
GGT ATT GAT AAG ATC ATT GTA GTC ACT GCC AAT GAT CCA TTT GTC AAT GCT GCT $\begin{array}{llllllllllllllllllllllllllll}G & I & D & K & I & I & V & V & T & A & N & D & P & F & V & N & A & A\end{array}$ TGG GGT AAA GCT TTA GGG TAT ACT GAT GAG GAG AAT TAT GTG GTA TTC GCT TCG

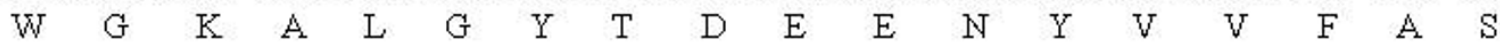
GAT GCT TAT GCT GAT TAC AGT AAG CAA TTA GGA GAT GGA TAC ACG GCT GAC TTC

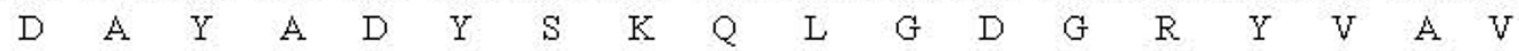
ACT AAA GGT GGA ATG GGT ATT AGA ACC GGA AGG TAT GTG GCT GTA ATT GAT GAT $\begin{array}{llllllllllllllllll}I & K & G & G & M & G & I & R & T & G & R & Y & V & A & V & I & D & D\end{array}$ GGG AAG ATT GTG TTT TCT GGA ACT GAA GAT AGT TTA GGA TAC ACT GAA ATT TCC $\begin{array}{llllllllllllllllll}G & K & I & V & F & S & G & T & E & D & S & L & G & Y & T & E & I & S\end{array}$ AGTGCT GAG ACC ATC CTT AAA CAA TTG TGA

$$
\begin{array}{llllllllll}
S & A & E & T & I & L & K & Q & L & *
\end{array}
$$

TACAACAATGGCAAGGTGGTCTAGTTTGTAGTATAGTCCATGGTCCATAGTGTGTACGTAT ATAGCCTAGTTGTATAAGTAATAAATTATCCAGCTGTCAAAAAAAAAAAAAAAAAAAAAA AAAAAAA

\section{Figure 2}

The full-lengthed nucleotide sequence of DhAHP and the predicted amino acid sequence. The start and stop codons ATG and TGA were boxed.

trast, only a small amount of the transcript was present in the control cell. Based on the differences in band intensity, it is evident that expression of DhAHP increased several fold only after $16 \mathrm{~min}$ of salt treatment. Thus, expression of the gene is rapidly induced by salt in $D$. hansenii.

The time course of induction of DhAHP by salt was further analyzed by relative quantification real-time RT-PCR. A small increase in DhAHP transcript was detected as early as 4 min upon salt ( $2.5 \mathrm{M} \mathrm{NaCl})$ treatment, but its expression was rapidly accelerated thereafter. Its level increased 1.9 and 2.9 fold over the control at 12 and 24 min, respectively, with the maximum induction of 8.0 to 12.1 fold occurring between 48 and $72 \mathrm{~min}$. After reaching its peak of expression at $72 \mathrm{~min}$, the transcript dropped off at 144 min (Fig. 5).

\section{Silencing by RNA interference and overexpression of DhAHP in D. hansenii}

To assess the effect of loss-of-function and gain-of-function of DhAHP on salt tolerance of D. hansenii, the silencing and overexpression transformants were examined for their ability to grow on YM11 medium containing $2.5 \mathrm{M}$ and $3.5 \mathrm{M} \mathrm{NaCl}$, respectively. As demonstrated by realtime PCR, the RNAi transformant of $D$. hansenii exhibited reduced expression of DhAHP transcript in the presence of $2.5 \mathrm{M} \mathrm{NaCl}$, relative to its wild type strain (Fig. 6A). Without any salt, both wild type strain and RNAi transformant showed a normal growth trend over $60 \mathrm{~h}$ (Fig. 6B). How- 


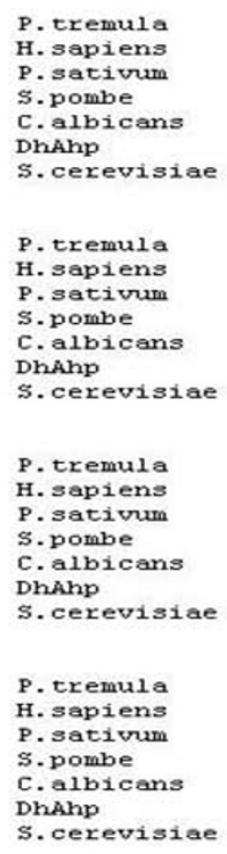

A

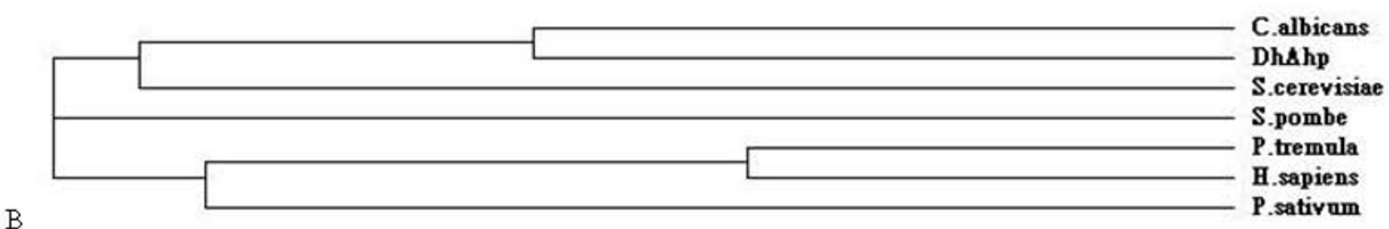

\section{Figure 3}

A. Multiple alignment of related sequences to DhAhp. The alignment was performed using the software of CLUSTAL W program http://www.ebi.ac.uk/Tools/clustalw2/index.html. Asterisks indicate identical amino acids and periods show conserved amino acid substitutions. Percent of overall identity similarity (in parentheses): I. DhAhp; 2. AHPI of S. cerevisiae (SwissProt: P380I3) (30\%); 3. PMP20 of S. pombe (Swiss-Prot: OI43I3) (33\%); 4. AHPII of C. albicans (Swiss-Prot: Q5AF44) (58\%); 5. peroxiredoxin of $P$. tremula (Swiss-Prot: Q8S3L0) (34\%); 6. peroxiredoxin of $P$. sativum (Swiss-Prot: B3GV28) (37\%); 7. peroxiredoxin of $H$. sapiens (Swiss-Prot: P30044) (25\%). Cys54, conserved in all Prxs, is identified as the peroxidative cysteine. B. The phylogenetic relationship between DhAhp and peroxiredoxin from other organisms.

ever, growth of the RNAi transformant was severely inhibited by $2.5 \mathrm{M} \mathrm{NaCl}$.

The overexpression transformant of $D$. hansenii had much higher AHP expression levels than its wild type counterpart when grown under $3.5 \mathrm{M} \mathrm{NaCl}$ and in the presence of the inducer methanol (Fig. 7A). Without any salt the overexpression trasnsformant showed a comparable growth to that of the wild type strain with or without the presence of methanol in the culture media (Fig. 8). Growth of both the wild type strain and the overexpression transformant was inhibited by $3.5 \mathrm{M} \mathrm{NaCl}$ (Fig. 8B). However, only the overexpression transformant showed enhanced growth in the presence of the inducer methanol. Thus, overexpression and suppression of $D h A H P$ reduce the salt tolerance of $D$. hansenii, respectively. The small enhancements in growth in the overexpression transformant under high salt, as compared to the wild type strain, is expected as expression of endogenous DhAHP can be largely induced by salt in this halophilic organism (Fig. 5).

\section{Overexpression of DhAHP in S. cerevisiae and $\mathbf{P}$. methanolica}

The function of DhAHP was further tested by overexpression of the gene in the two salt-sensitive yeasts $S$. cerevisiae and P. methanolica. As expected, the levels of DhAHP transcript in the wild type strains of the two species were very low even under high salt conditions, but its expression levels in the overexpression transformants increased drastically, especially in the presence of the inducer methanol 

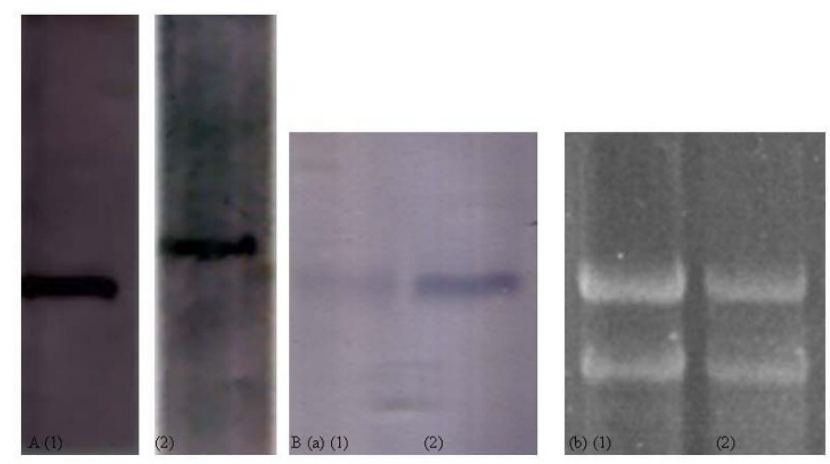

\section{Figure 4}

A. Southern blot showing a single restriction fragment of $\boldsymbol{D}$. hansenii. Approximately $20 \mu \mathrm{g}$ total DNA was digested to completion with EcoRI (lane I) or BamHI (lane 2), electrophoresed on agarose gel, transferred to nylon membrane and hybridized to DhAHP probe. B. Northern blot of DhAHP transcript as affected by salt treatment. Total RNA was isolated and electrophoresed on agarose-formaldehyde gel, transferred to nylon membrane and hybridized to DhAHP probe (A). The gel was stained with ethidium bromide prior to blotting $(B)$. Lane I and 2 indicate RNAs extracted from $D$. hansenii cells after inducted by $2.5 \mathrm{M} \mathrm{NaCl}$ for 0 and $16 \mathrm{~min}$, respectively.

(Figs. 7B, 7C). The salt tolerance of the overexpression transformants of the two yeasts was evaluated by culture in YPD medium containing $2.0 \mathrm{M} \mathrm{NaCl}$ for $S$. cerevisiae (Fig. 9b) and in YPAD medium containing $2.5 \mathrm{M} \mathrm{NaCl}$ for $P$. methanolica, relative to those of their wild type counterparts (Fig. 10b). For both species, the wild type strain and the overexpression transformant showed a normal and comparable growth over time when they were grown in

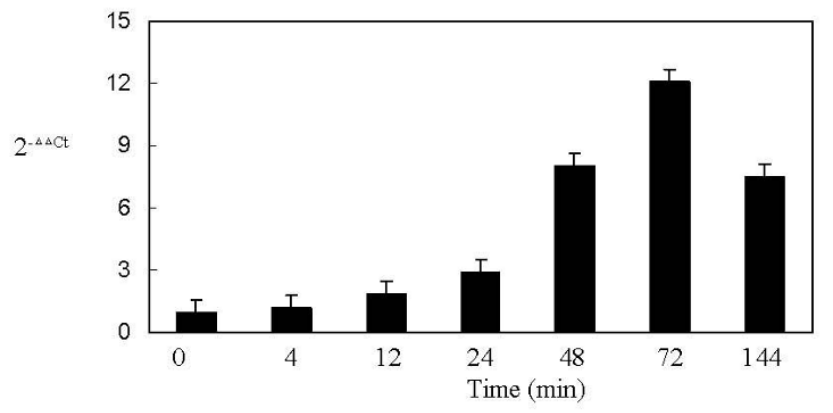

Figure 5

Time course of induction of DhAHP transcript by $\mathbf{2 . 5}$ $\mathbf{M ~ N a C l}$, as determined by real-time RT-PCR. Its transcript level increased I.3, I.9, 2.9, 8.0, I2.I and 6.I fold after $4,12,24,48,72$ and 144 min of induction, respectively. Data presented were means +/- S.D. from 3-4 replicates of measurement.

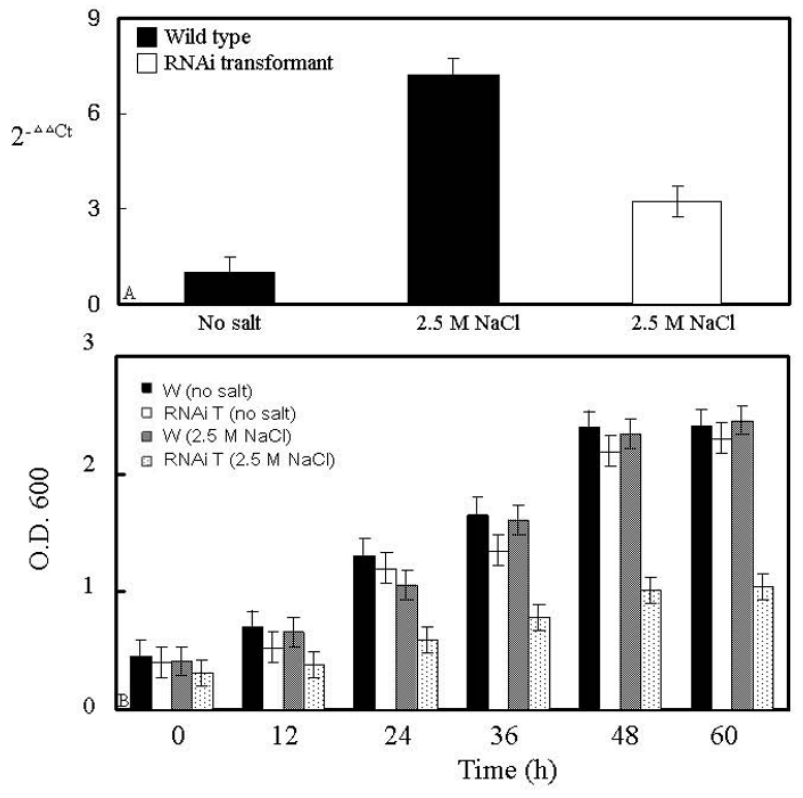

Figure 6

(A) Relative levels of DhAHP transcript of D. hansenii and its RNAi transformant as affected by salt. Cells were grown on YMI I media containing $2.5 \mathrm{M} \mathrm{NaCl}$ for 72 min, and their DhAHP transcripts determined by real-time RT-PCR. (B) Growth of D. hansenii and its DhAHP RNAi transformant. Cells were grown on YMII media with or without $2.5 \mathrm{M} \mathrm{NaCl}$. W: wild type strain, RNAi T: RNAi transformant. Data presented were means +/- S.D. from 3-4 replicates of measurement.

media without containing salt in the presence or absence of methanol (Figs. 9a, Fig. 10a). In contrast, growth of the wild type strains of these salt-sensitive species was largely inhibited by high salt (Figs. 9b, Fig. 10b). However, only the overexpression transformants were able to maintain substantial growth under high salt, especially in the presence of methanol. The degrees of enhancement in salt tolerance by overexpression of DhAHP were more significant in S. cerevisiae and in P. methanolica (Figs. 9b, 10b) than in D. hansenii (Fig. 8b). The results indicate that overexpression of DhAHP confers enhanced salt tolerance to both salt sensitive $S$. cerevisiae and $P$. methanolica, allowing them to be able to grow at higher salt levels than they can normally tolerate.

\section{Intracellular ROS}

To see if the enhanced salt tolerance by overexpression of DhAHP in the three yeast species was due to reduced oxidative stress, the cellular ROS level was determined after the cells were grown under high $\mathrm{NaCl}$ conditions $(3.5 \mathrm{M}$ for $D$. hansenii, 2.0 $\mathrm{M}$ for $S$. cerevisiae and $2.5 \mathrm{M}$ for $P$. methanolica) for $5 \mathrm{~h}$. As shown in Fig.11A-C, $\mathrm{NaCl}$ 

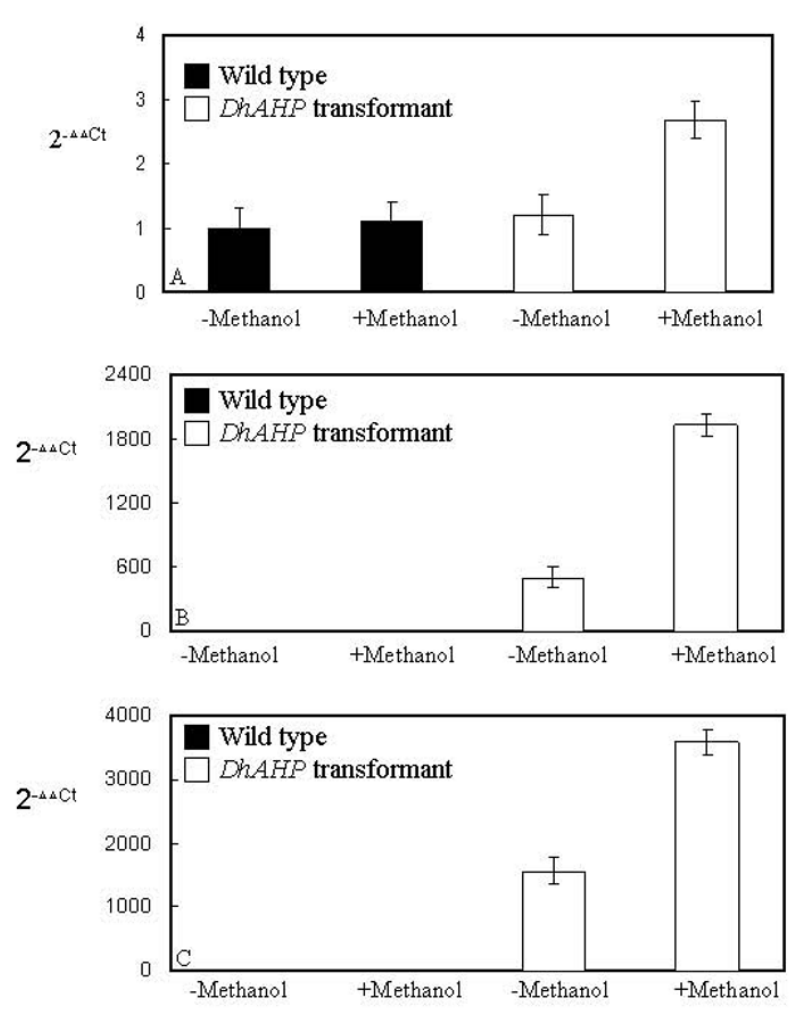

\section{Figure 7}

Relative levels of DhAHP transcript of three yeasts and their DhAHP overexpression transformants. Cells of D. hansenii (A), S. cerevisiae (B) and P. methanolica (C) were grown in media containing 3.5, 2.0 and $2.5 \mathrm{M} \mathrm{NaCl}$, respectively, in the presence or absence of methanol for 72 min, and their DhAHP transcripts determined by real-time RT-PCR. For each species, the level for the wild type strain grown in media without methanol was taken as I. Since the wild type strains of S.c. and P.m do not contain DhAHP their DhAHP transcript levels were low while their overexpression transformants showed high levels of expression relatively. Data presented were means $+/$ S.D. from 3-4 replicates of measurement.

induced accumulation of ROS in the wild type strains of the three yeast species, and the addition of methanol further increased its accumulation. It is also noticeable that the increases in ROS accumulation under high salt were much greater in S. cerevisiae and $P$. methanolica than in $D$. hansenii. The DhAHP overexpression transformants of the three species also exhibited a similar trend towards salt and methanol treatments but the amounts of ROS accumulated were considerably lower than those of their wild type counterparts. The reduction in ROS accumulation was more significant upon methanol induction, especially in the overexpression transformants of $S$. cerevisiae and $P$. methanolica. These results, correlated well with the data on levels of DhAHP expression (Fig. 7A-C) and on growth

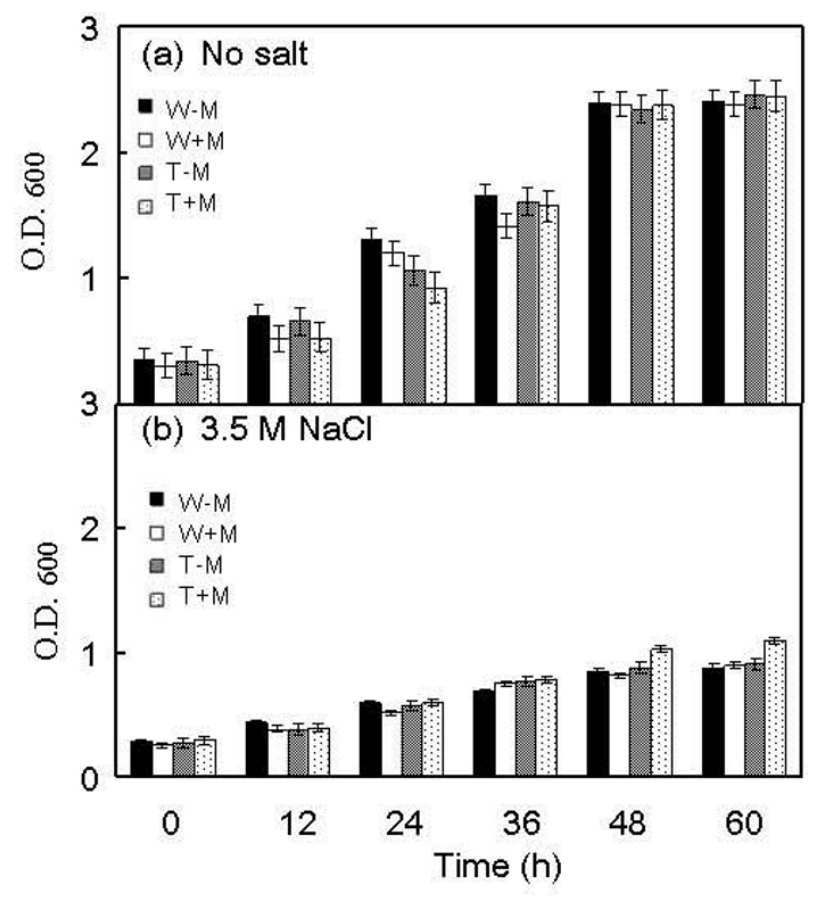

Figure 8

Growth of D. hansenii and its DhAHP overexpression transformant as affected by salt. Cells were cultured in YMI I media with or without $3.5 \mathrm{M} \mathrm{NaCl}$ and in the presence or absence of methanol for 5 days. W-M: wild type strain, without methanol, $W+M$ : wild type strain, with $0.5 \%$ methanol, T-M: transformant, without methanol, T+M: transformant with $0.5 \%$ methanol. Data presented were means +/- S.D. from 3-4 replicates of measurement.

(Figs. 8, 9, 10), indicate that expression of DhAHP in these yeasts can lead to enhanced salt tolerance by reducing the level of accumulated ROS via DhAhp. Thus, the higher sensitivities of $S$. cerevisiae and $P$. methanolica to salt, relative to $D$. hansenii, may be associated with their inability to scavenge ROS.

\section{Discussion}

Organisms are constantly exposed to various stresses, which cause considerable reduction in growth. In adaptation, organisms respond to stress through a number of physiological and developmental changes. Thus, expression of many genes is altered in such responses. Identification of the particular gene or genes responsible for the specific adaption to such stimuli is a major challenge in modern biology; it requires methods which rapidly and efficiently compare the transcripts expressed in the organism subject to stress. An equalizing cDNA subtraction hybridization method provides the technical basis for such a comparison. It has been demonstrated successfully to clone a number of differentially expressed genes [27]. 


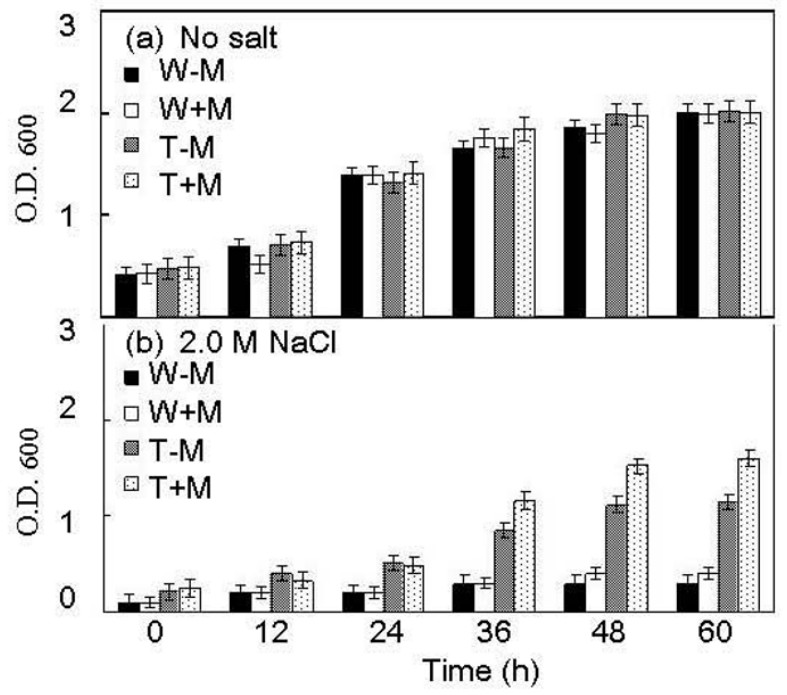

Figure 9

Growth of S. cerevisiae and its DhAHP overexpression transformant as affected by salt. Cells were cultured on YPD media with or without $2.0 \mathrm{M} \mathrm{NaCl}$ and in the presence or absence of methanol for 5 days. W-M: wild type strain, without methanol, $\mathrm{W}+\mathrm{M}$ : wild type strain, with $0.5 \%$ methanol; T-M: transformant, without methanol; $T+M$ : transformant with $0.5 \%$ methanol. Data presented were means +/- S.D. from 3-4 replicates of measurement.

Isolation of differentially expressed genes in the extremely halophilic yeast $D$. hansenii would serve as an initial step towards understanding its tolerance mechanisms against salinity.

\section{Salt-induced genes in D. hansenii}

As discussed in the Background section, a number of saltrelated genes have been identified in the extremely halophilic yeast $D$. hansenii. As expected, most of the saltupregulated genes identified so far are involved in osmoregulation or transport of ions. By using forward subtractive hybridization, we have identified, cloned and sequenced $D h A H P$, a new salt induced gene, from $D$. hansenii by applying salt stress. Further characterization of the functional role of the gene will aid to our understanding of the underlying halotolerance mechanisms in this halophilic yeast.

\section{Characterization of salt-induced DhAHP and its protein}

High salinity, which is caused typically by $\mathrm{NaCl}$, results in ion toxicity and hyperosmotic stress leading to numerous secondary pathological effects including generation of ROS [28] and programmed cell death. It's not surprising that one of the major upregulated genes under salinity stress, DhAHP, is orthologous to the alkyl hydroperoxide

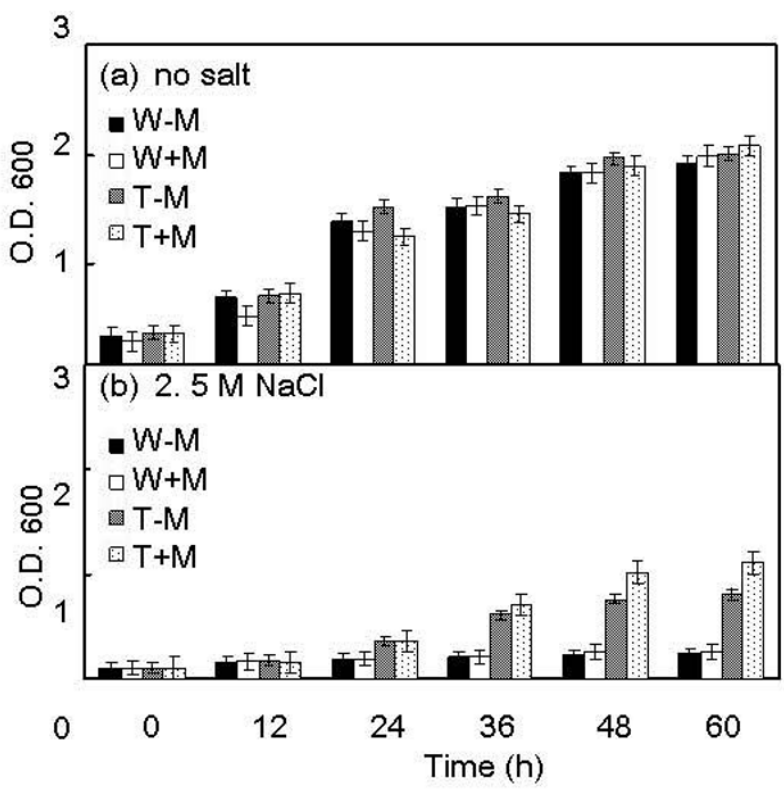

Figure 10

Growth of $P$. methanolica and its DhAHP overexpression transformant as affected by salt. Cells were cultured in YPAD media with or without $2.5 \mathrm{M} \mathrm{NaCl}$ and in the presence or absence of methanol for 5 days. W-M: wild type strain, without methanol, $W+M$ : wild type strain, with $0.5 \%$ methanol; T-M: transformant, without methanol; T+M: transformant with $0.5 \%$ methanol. Data presented were means + / - S.D. from 3-4 replicates of measurement.

reductase of the peroxiredoxin family. Ahp is a member of the peroxiredoxin family of enzymes, which possess activity against $\mathrm{H}_{2} \mathrm{O}_{2}$, organic peroxides, and peroxynitrite [18]. DhAHP has not been previously described for its role in salt tolerance in D. hansenii. Comparison of protein sequences showed that DhAhp shares a high similarity to Ahp11 of the yeast $C$. albicans. Multiple sequence alignment analysis of Ahps showed the protein from $D$. hansenii has a high similarity to that of C. albicans (Fig. 3A) and phylogenetic analysis revealed that Ahp of $D$. hansenii is more closely related to the yeast than to the plant or mammalian peroxiredoxins (Fig. 3B). Thus, DhAhp belongs to the alkyl hydroperoxide reductase of the peroxiredoxin family. Previously, Kurtzman and Robnett [29] have suggested that $D$. hansenii is phylogenetically related to $C$. albicans based on the fact that they are both ascomycetous yeasts. The high similarity between the Ahps from both species further supports this notion. In addition, both organisms use an alternative genetic yeast code in which the CUG codon may be used as a serine codon [30]. Taken together, these results suggest that DhAhp and C. albicans Ahp11 have common ancestry, but show divergent evolution. 

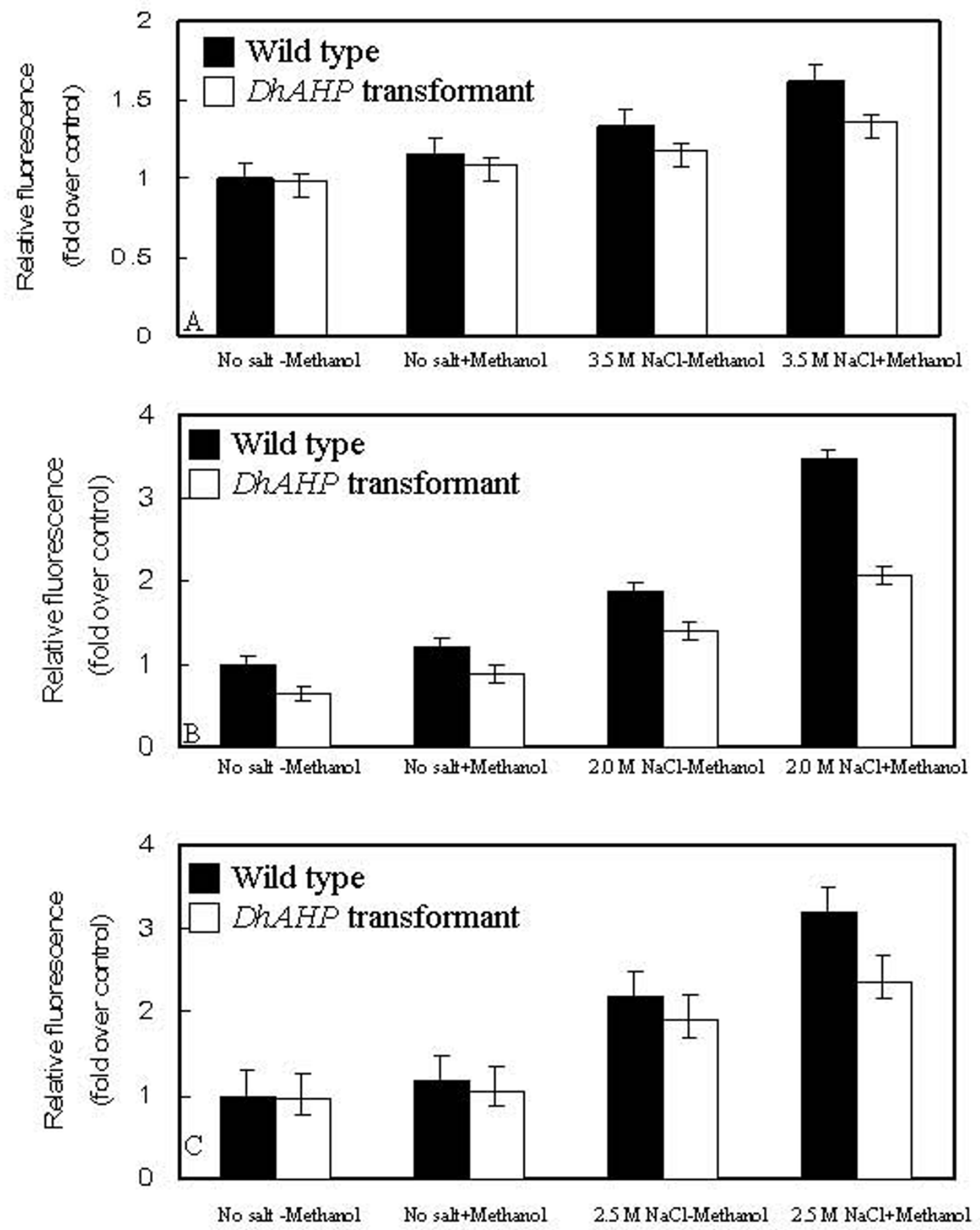

Figure I I

Cellular ROS levels of three yeasts and their DhAHP overexpression transformants as affected by salt. Cells of $D$. hansenii $(\mathbf{A})$, S. cerevisiae $(\mathbf{B})$ and $P$. methonolica $(\mathbf{C})$ were grown in liquid media with or without salt and in the presence or absence of $0.5 \%$ methanol for $5 \mathrm{~h}$. ROS levels, as measured by fluorescence signal, were presented as relative values. Data presented were means +/- S.D. from 3-4 replicates of measurement. 
The closest structural homolog to DhAHP is the PrxD (Type Ii) of Populus tremula (PDB:1TP9A) (data not shown), which contains two cysteine residues. Though poplar Prx contains two conserved cysteine residues, it is assumed to function as a 1-Cys Prx because site-directed mutagenesis has demonstrated that only the catalytic cysteine of the poplar Prx is essential for hydroperoxide reduction [31]. Previously, the type II TPx from S. cerevisiae was reported to contain three Cys residues at positions 31, 62 and 120, and its disulfide linkage is between 62 and 120 and Cys-31 has no effect on TPx activity [32]. Though structural and sequence analyses of the deduced protein indicate that DhAhp contains 2 Cys residues at positions 24 and 54, the multiple sequence alignment of Ahps identifies the conserved Cys-54 as the peroxidative cysteine (Fig. 3). The role of Cys-24 in D. hansenii Ahp remains to be explored in the future. Therefore, DhAhp is clearly a member of the disulfide oxidoreductases and can be considered a 1-Cys Prx.

\section{Regulation of expression of DhAHP}

Alkyl hydroperoxide reductases have been identified previously as oxidative stress proteins in Salmonella typhimurium [33] and Bacillus subtilis [23] and their expression is known to be upregulated by oxidative factors. However, the finding of an extensive accumulation of Ahp in the halophilic yeast $D$. hansenii by salt is reported for the first time in this study. Consistently, overexpression of $D$. hansenii Ahp in D. hansenii (Fig. 7) and in the two salt-sensitive yeasts S. cerevisiae and P. methanolica (Fig. 8 and 9) further increases their tolerance to salt. On the contrary, suppression of its expression in $D$. hansenii resulted in a lower tolerance to salinity (Fig. 6). Clearly, the results suggest that $D h A H P$ is induced by salt and its expression confers the high salt tolerance in $D$. hansenii. A previous study also revealed that the expression of a homolog to the Escherichia coli Ahp is induced by osmotic shock in Staphylococcus aureus [34]. Similarly, the expression of Ahp in Shewanella putrefaciens is accompanied by accumulation of the corresponding transcript under $\mathrm{NaCl}$ stress [35] and the activity of the alkyl hydroperoxidase enzyme is dependent on salt concentration [36]. Collectively, the results from these studies indicate that expression of Ahps in general is upregulated not only by oxidative factors but also by other stresses, such as drought and salinity.

Hydrogen peroxide level is known to increase within the cell in response to various stress factors and act as an intracellular messenger for induction of genes related to defense against oxidative environments [37]. Treatment of cells with hydrogen peroxide mimics stress and induces defense signaling by activating mitogen-activated protein kinase and stimulates cell growth [38]. The ROS levels of $D$. hansenii, S. cerevisiae and P. methanolica also increase in response to salt and methanol treatments, and the degrees of increase are more pronounced in the two salt-sensitive yeast species than the halophilic $D$. hansenii (Fig. 11). Furthermore, the DhAHP overexpression transformants of these species have reduced amounts of ROS accumulated than their wild type strains, indicating the protective role of Ahp. These results are in agreement with the earlier observations that Ahp genes play an important role in peroxide resistance in Bacillus subtilis [23], Clostridium pasteurianum [24], Burkholderia cenocepacia [25], Shewanella putrefaciens [35] and Porphyromonas gingivalis [39] under various stress conditions (e.g. hydrogen peroxide, high/ low temperature and high/low $\mathrm{pH}$ ). Therefore, the induced expression and accumulation of DhAhp in saline environments to detoxify ROS is a very important survival mechanism for this halophilic organism.

\section{Conclusion}

In summary, the Ahp gene isolated from the extremely halophilic yeast $D$. hansenii under salt stress in this study is a new gene relative to its salt tolerance mechanism. It is rapidly induced and accumulates to large quantities in $D$. hansenii to reduce accumulation of ROS. Molecular characterization shows that DhAhp, a cytosolic protein, belongs to the alkyl hydroperoxide reductase of the 1-Cys type peroxiredoxin family. The DhAhp and C. albicans Ahp11 have a common ancestry but show divergent evolution. Silencing of its expression by RNA interference resulted in decreased tolerance to salt stress. On the other hand, overexpression of the DhAHP in D. hansenii and the two salt-sensitive yeasts $S$. cerevisiae and $P$. methanolica conferred enhanced tolerance to salt with reduced accumulation of ROS. Clearly, the multiple activities (peroxidase, chaperone, redox signaling) possessed by Ahps are essential for its central role in protecting the cellular metabolism of yeast against ROS built-up under stress conditions. Compared with the two salt-sensitive yeasts, the extreme halotolerance exhibited by $D$. hansenii may be due to its ability to scavenge ROS by Ahp. Thus, the results of this study contribute to our understanding of the underlying mechanisms by which the extremely halophilic yeast $D$. hansenii adapts to high salt. Manipulation of antioxidant enzymes in industrial microorganisms, as demonstrated in S. cerevisiae and P. methanolica in this study, or crops may bring about enhanced growth and production of useful products under adverse culture conditions. Overexpressing enzymes involved in redox reaction in crops, such as superoxide dismutase [40] and glutathione peroxidase [41] has resulted in enhanced tolerance to salt and other stress.

\section{Methods \\ Yeast strains and growth conditions}

The yeast strains used in this work included $D$. hansenii strain BCRC No. 21947, isolated from Hsilo County, Taiwan, S. cerevisiae Neo Type strain Y1 BCRC No. 21447 
from brewer's top yeast, obtained from FIRDI (Food Industry Research and Development Institute, Hsin-chu City, Taiwan), and P. methanolica strain PMAD11 genotype ade2-11, obtained from Invitrogen, U.S.A. D. hansenii was cultured at $24^{\circ} \mathrm{C}$ in YM medium $(0.3 \%$ yeast extract, $0.3 \%$ malt extract, $0.5 \%$ peptone, $1 \%$ dextrose) while $S$. cerevisiae and $P$. methanolica were cultured at $28^{\circ} \mathrm{C}$ in YPD medium ( $1 \%$ yeast extract, $2 \%$ peptone, $2 \%$ dextrose) and YPAD medium ( $1 \%$ yeast extract, $2 \%$ peptone, $2 \%$ dextrose, $0.01 \%$ adenine), respectively.

\section{RNA extraction and poly $\left(A^{+}\right)$RNA purification}

Total RNA was extracted with a modified hot phenol protocol [42]. Poly $\left(\mathrm{A}^{+}\right)$RNA was isolated from total RNA using Mag-Net mRNA Isolation Kit according to the manufacturer's instruction (Amresco, Inc. USA). Concentration of RNA was determined using a NanoDrop spectrophotometer (NanoDrop, Wilmington, USA). RNA quality was verified by electrophoresis on $1.5 \%$ formaldehyde agarose gel and stained with ethidium bromide.

\section{Subtractive hybridization and construction of subtracted cDNA library}

Subtractive hybridization was performed using PCR-select cDNA Subtraction Kit (Clontech, Palo Alto, CA, U.S.A.). For screening of differentially upregulated genes, cDNA synthesized from the $2.5 \mathrm{M} \mathrm{NaCl}$ treated yeast cells for 24 min was used as the tester while that from non-treated cells served as the driver. The PCR products of forward subtraction were subcloned into the pGEMR-T Easy Vector (Promega, USA). Competent cells of E. coli (XL-Blue) was transfected with the plasmids and grown on LB-agar medium containing 5-bromo-4-chloro-3-indolyl-b-dgalactoside (X-gal) (Sigma, U.S.A.), isopropyl $\beta$-D-1-thiogalactopyranoside (IPTG) (Sigma, U.S.A.) and ampicillin. Individual white colonies with insert DNA were randomly picked for further analysis.

\section{Sequencing and sequence analysis}

White clones from the forward subtractive hybridization libraries were sequenced with the universal T7 or SP6 sequencing primers using an automatic DNA sequencer (3100 Genetic Analyzer, ABI, U.S.A). All inserted sequences were queried for similarity through the NCBI database using BLASTX sequence comparison software http://www.ncbi.nlm.nih.gov/BLAST.

\section{Quantification of DhAHP by quantitative real-time PCR (Q-RT-PCR)}

Total RNA isolated from yeast cells treated with $\mathrm{NaCl}$ for various time intervals was first treated with DNase I (Promega, U.S.A.) to remove DNA contamination before cDNA synthesis [43]. cDNA was synthesized using High Capacity cDNA Reverse Transcription Kit (P/N 4368814,
ABI, U.S.A.) for RT-PCR according to the manufacturer's instruction. The sequence forward and reverse primers for Q-RT-PCR were designed using the primer Express ${ }^{\mathrm{R}}$ Software provided by Applied Biosystems. A set of D. hansenii $18 \mathrm{~S}$ ribosomal RNA primers was designed for use as an endogenous control.

\section{$18 \mathrm{~S}$ forward: G'-CGTCCCTGCCCTTTGTACAC-3'}

18 reverse: G5'-GCCTCACTAAGCCATTCAATCG-3'

DhAHP target forward: G5'-GGAGCCCCAGGAGCATTTA$3^{\prime}$

DhAHP target reverse: G5'-TGGGCCAAATAATCGGGAAT$3^{\prime}$

Real-time PCR assay was carried out in an ABI PRISM 7500 Sequence Detection System (ABI, U.S.A.). The amplification of the target genes was monitored every cycle by SYBR-Green fluorescence.

\section{Rapid amplification of CDNA ends (RACE)}

The full-lengthed cDNA clone of DhAHP was obtained by rapid amplification of the CDNA ends using the GeneRacerTM Kit (Invitrogen, U.S.A.), as described in the manual provided by the manufacturer. The forward and reverse gene specific primers (GSPs) used for RACE were designed based on the DhAHP cDNA sequence. The universal primers for 5' and 3' Race were GeneRace 5' and GeneRace 3', respectively, provided in the kit. After PCR the DNA fragments were cloned into pGEMR-T Easy vector (Promega, U.S.A.) for sequencing.

Forward (GSP): 5'- GTCAATGCTGCTTGGGGTAAAG CTTTA-3'

Reverse (GSP):5'- GGTCTCAGCACTGGAAATTTCAGTG$3^{\prime}$

GeneRace 5':5'- CGACTGGAGCACGAGGACACTGA-3'

\section{GeneRace 3':5'- GCTGTCAACGATACGCTACGTAACG-3'}

\section{Bioinformatics analysis}

The deduced amino acid sequence of DhAHP was analyzed with the Expert Protein Analysis System http:// www.expasy.org/. Multiple sequence alignment was performed for sequence comparison and alignment of $D$. hansenii Ahp and two other reported AHPs (Swiss-Prot: P38013 and Q5AF44) from S. cerevisiae and C. albicans and peroxisomal membrane protein (Swiss-Prot: O14313) from $S$. pombe and three other structural homolog proteins (Swiss-Prot:Q8S3L0, B3GV28 and 


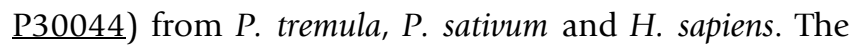
alignment and phylogenetic analysis were carried out by the protein sequence alignment program CLUSTAL W.

\section{Southern and northern hybridization analysis}

Genomic DNA was isolated from yeast cells by the method of Hoffman and Winston [44]. Southern and northern hybridization analyses were performed using the DIG High Prime DNA Labeling and Detection Starter Kit (Roche Diagnostics, Switzerland). For Southern hybridization, $20 \mu \mathrm{g}$ genomic DNA was digested with EcoRI and BamHI and electrophoretically separated on $0.7 \%(\mathrm{w} / \mathrm{v})$ agarose gels in TBE buffer and DNA fragments blotted onto nylon membrane (Amersham Pharmacia Biotech, U.K.) by $20 \times$ SSC. The full-lengthed DhAHP DNA was labeled and used as a hybridization probe. For nothern hybridization analysis, RNA was extracted from $D$. hansenii that was not treated or treated with $2.5 \mathrm{M} \mathrm{NaCl}$ for 16 min, separated by electrophoresis in formaldehyde gels, blotted onto nylon membrane and hybridized with labeled DhAHP DNA, as described above for Southern analysis.

\section{Silencing of DhAHP expression in D. hansenii by RNA interference}

To test the function of DhAHP, RNA interference was employed to suppress its expression in D. hansenii using the Knockout RNAi System Kit (Clontech, U.S.A.), as described in the manual by the manufacturer. The oligonucleotide sequences including BamHI and EcoRI sites, target sense sequence, hairpin loop, target antisense sequence and terminator were shown as follow.

BamHI Target sense sequence Hairpin loop Target antisense sequence Terminator

\section{5'-GATTCGACATATTMLCGATTATTTGTTCAAGA- GACAAATAATCGGGAATATGTTTTTTTG-3'}

\section{3'-GCTGTATAAGGGCTAATAAACAAGTTCTCTGTTTATT- AGCCCTTATACAAAAAAACTTAA-3' EcoRI}

A chemical method based on $\mathrm{LiCl}$, as described by Tarutina and Tolstorukov [45], was used to transfect D. hansenii and the RNAi transformant was screened by its poor ability to grow on YM11 solid media containing $2.5 \mathrm{M}$ $\mathrm{NaCl}$. The transformant was confirmed by sequencing the introduced DNA fragment in the genome with specific primers and by Q-RT-PCR.

\section{Overexpression of DhAHP in D. hansenii, S. cerevisiae and $P$. methanolica}

To further test its functional role in relation to salt tolerance, DhAHP was overexpressed in three yeast species with contrasting degrees of salt tolerance. The entire ORF of DhAHP was first amplified by PCR utilizing the overexpression 5 ' primer, which introduced an EcoRI site in front of the starting ATG codon, and the overepression 3' primer, which introduced a BamHI site before the stop codon. This DNA fragment was inserted into the expression vector of pMETB (Invitrogen, U.S.A.). The plasmid DNA of the DhAHP/pMETB veector was digested with Pst I to release the $P_{A U G 1} / D h A H P$ expression cassette, which was then introduced into $D$. hansenii, $S$. cerevisiae and $P$. methanolica by a chemical method based on $\mathrm{LiCl}$, as described by Tarutina and Tolstorukov [45]. The AUG1 sequence is a methanol inducible promoter to drive the expression of introduced DhAHP. Functional complementation was used to screen transformants from the three species by culture on solid media containing $0.5 \%$ methanol and higher $\mathrm{NaCl}$ concentrations than they can normally tolerate. For isolation of $D$. hansenii overexpression transformants YM medium containing $3.5 \mathrm{M} \mathrm{NaCl}$ was used, for $S$. cerevisiae transformants YPD medium containing $1.5 \mathrm{M} \mathrm{NaCl}$ was used and for $P$. methanolica transformants YPAD medium containing $2.0 \mathrm{M} \mathrm{NaCl}$ was adopted. The transformants were confirmed by sequencing the $\boldsymbol{P}_{\text {AUG1 }}$ DNA fragment in the genome with specific primers and by Q-RT-PCR with cells grown under high salt in the presence or absence of methanol. The ability of the selected transformants to tolerate salt was further assessed by growing in liquid media containing high $\mathrm{NaCl}$ concentrations.

\section{Measurement of intracellular ROS}

For measurement of cellular ROS, the redox-sensitive fluorescent probe 2', 7'-dihydrodichlorofluorescein diacetate (DCFA-DA) (Sigma, U.S.A.) was used according to Chattopadhyay et al. [46]. The cells of wild type strains and DhAHP overexpression transformants were grown in appropriate liquid media without any salt for approximately $36 \mathrm{~h}$ (1 O.D. at $600 \mathrm{~nm}$ ) and switched to fresh media containing high $\mathrm{NaCl}(3.5 \mathrm{M}$ for D. hansenii, $2.0 \mathrm{M}$ for $S$. cerevisiae and $2.5 \mathrm{M}$ for $P$. methanolica) with or without methanol for $5 \mathrm{~h}$. To determine ROS, cells were harvested by centrifugation and treated with $10 \mu \mathrm{M}$ DCFA for $30 \mathrm{~min}$ at $30^{\circ} \mathrm{C}$. The cells were re-suspended and washed in water and extracted by vortexing with glass beads. Extracts were centrifuged and fluorescence in the supernatant was measured with $\lambda \mathrm{EX}=485 \mathrm{~nm}$ and $\lambda \mathrm{EM}=524 \mathrm{~nm}$ in a fluorescence spectrophotometer (Infinite F200). Fluorescence signals were expressed relative to that of the wild type strain before any stress treatments (fold over control).

\section{Authors' contributions}

HFC planned and designed the study, performed the experiments and analyzed the results and drafted the manuscript. YFY contributed equally. MSBK initiated and supervised the study, assisted in data analysis and revised 
the manuscript. All authors read and approved the final manuscript.

\section{Acknowledgements}

The authors acknowledge the supports of Tainan District Agricultural Improvement Station, Council of Agriculture, Taiwan Executive Yuan and the Graduate Institute of Agricultural Biotechnology, National Chiayi University. The authors also thank Emery M. Ku for critical reading of the manuscript.

\section{References}

I. Prista C, Almagro A, Loureiro-Dias MC, Ramos J: Physiological basis for the high salt tolerance of Debaryomyces hansenii. Appl Environ Microbiol 1997, 63:4005-4009.

2. Norkrans B: Studies on marine occurring yeasts: Growth related to $\mathrm{pH}, \mathrm{NaCl}$ concentration and temperature. Arch fur Mikrobiol 1966, 54:374-392.

3. Onishi H: Osmophilic yeasts. Advaces in Food Res 1963, 12:53-94.

4. Prista C, Loureiro-Dias MC, Montiel V, García R, Ramos J: Mechanisms underlying the halotolerant way of Debaryomyces hansenii. FEMS Yeast Res 2005, 5:693-70I.

5. Bressan RA, Bonnert HJ, Hasegawa M: Genetic engineering for salinity stress tolerance. In Advances in Plant Biochemistry and Molecular Biology. Bioengineering and Molecular Biology of Plant Pathways Volume I. Edited by: Bohner HJ, Nguyen H, Lewis NG. Pergaman Press; 2008:p374-384.

6. Neves ML, Oliveira RP, Lucas CM: Metabolic flux response to salt-induced stress in the halotolerant yeast Debaryomyces hansenii. Microbiol 1997, 143:1133-1139.

7. Almagro A, Prista C, Castro S, Quintas C, Madeira-Lopes A, Ramos J, Loureiro-Dias MC: Effects of salts on Debaryomyces hansenii and Saccharomyces cerevisiae under salt stress conditions. Intl J Food Microbiol 2000, 56:191-197.

8. Thomé-Ortiz PE, Penã A, Ramirez J: Monovalent cation fluxes and physiological changes of Debaryomyces hansenii grown at high concentrations of $\mathrm{KCl}$ and $\mathrm{NaCl}$. Yeast 1998, | 4:|355-|37|.

9. Calderón-Torres M, Peña A, Thomé PE: DhARO4, an amino acid biosynthetic gene, is stimulated by high salinity in Debaryomyces hansenii. Yeast 2006, 23:725-734.

10. Bansal PK, Mondal AK: Isolation and sequence of the HOG I homologue from Debaryomyces hansenii by complementation of the hog I delta strain of Saccharomyces cerevisiae. Yeast 2000, |6:8|-88.

II. Almagro A, Prista C, Benito B, Loureiro-Dias MC, Ramos J: Cloning and expression of two genes coding for sodium pumps in the salt-tolerant yeast Debaryomyces hansenii. J Bacteriol 200I, I 83:325I-3255.

12. Gori K, Hebraud M, Chambon C, Mortensen HD, Arneborg N, Jespersen L: Proteomic changes in Debaryomyces hansenii upon exposure to $\mathrm{NaCl}$. FEMS Yeast Res 2007, 7:293-303.

13. Montiel V, Ramos J: Intracellular $\mathbf{N a}$ and $\mathrm{K}$ distribution in Debaryomyces hansenii. Cloning and expression in Saccharomyces cerevisiae of DhNHXI. FEMS Yeast Res 2007, 7:102-109.

14. Carcia-Salcedo R, Montiel V, Calero F, Ramos J: Characterization of DhKHAl, a gene coding for a putative $\mathrm{Na}+$ transporter from Debaryomyces hansenii. FEMS Yeast Res 2007, 7:905-9II.

15. Demasi AP, Pereira GA, Netto LE: Yeast oxidative stress response: Influences of cytosolic thioredoxin peroxidase I and of the mitochondrial functional state. FEBS J 2006, 273:805-816.

16. Storz G, Christman MF, Sies H, Ames BN: Spontaneous mutagenesis and oxidative damage to DNA in Salmonella typhimurium. Proc Natl Acad Sci USA 1987, 84:8917-8921.

17. Jamieson DJ: Oxidative stress responses of the yeast Saccharomyces cerevisiae. Yeast 1998, 14:15 II-1527.

18. Knoops B, Loumaye E, Eecken V Van Der: Evolution of the peroxiredoxins. Subcell Biochem 2007, 44:27-40.

19. Hofmann B, Hecht HJ, Flohé L: Peroxiredoxins. Biol Chem 2002, 383:347-364.

20. Wood ZA, Schroder E, Harris JR, Poole LB: Structure, mechanism and regulation of peroxiredoxins. Trends Biochem Sci 2003, 28:32-40.
21. Tartaglia LA, Storz G, Brodsky MH, Lai A, Ames BN: Alkyl hydroperoxide reductase from Salmonella typhimurium. Sequence and homology to thioredoxin reductase and other flavoprotein disulfide oxidoreductases. J Biol Chem 1990, 265: $10535-10540$.

22. Poole LB, Ellis HR: Flavin-dependent alkyl hydroperoxide reductase from Salmonella typhimurium. I. Purification and enzymatic activities of overexpressed AhpF and AhpC proteins. Biochem 1996, 35:56-64.

23. Bsat N, Chen L, Helmann JD: Mutation of the Bacillus subtilis alkyl hydroperoxide reductase (ahpCF) operon reveals compensatory interactions among hydrogen peroxide stress genes. J Bacteriol 1996, 1 78:6579-86.

24. Reynolds C, Michael J, Poole LB: An NADH-dependent bacterial thioredoxin reductase-like protein in conjunction with a glutaredoxin homologue form a unique peroxiredoxin (AhpC) reducing system in Clostridium pasteurianum. Biochem 2002, 41: |990-200|.

25. Chung JW, Speert DP: Proteomic identification and characterization of bacterial factors associated with Burkholderia cenocepacia survival in a murine host. Microbiol 2007, 153:206-14.

26. Loprasert S, Sallabhan R, Whangsuk W, Mongkolsuk S: Compensatory increase in ahpC gene expression and its role in protecting Burkholderia pseudomallei against reactive nitrogen intermediates. Arch Microbiol 2003, 180:498-502.

27. Jiang H, Lin JJ, Su ZZ, Goldstein NI, Fisher PB: Subtraction hybridization identifies a novel melanoma differentiation associated gene, mda-7, modulated during human melanoma differentiation, growth and progression. Oncogene 1995, I I:2477-2486.

28. Gueta-Dahan Y, Yaniv Z, Zilinskas A, Ben-hayyinm G: Salt and oxidative stress: similar and specific responses and their relation to salt tolerance in Citrus. Planta 1997, 203:460-469.

29. Kurtzman CP, Robnett C): Identification and phylogeny of ascomycetous yeasts from analysis of nuclear large subunit (26S) ribosomal DNA partial sequences. Antonie Van Leeuwenhoek 1998, 73:331-37I.

30. Tekaia F, Blandin G, Malpertuy A, Llorente B, Durrens P, ToffanoNioche C, Ozier-Kalogeropoulos O, Bon E, Gaillardin C, Aigle M, Bolotin-Fukuhara $M$, Casarégola $S$, de Montigny J, Lépingle $A$, Neuvéglise $C$, Potier $S$, Souciet J, Wésolowski-Louvel M, Dujon B: Genomic exploration of the hemiascomycetous yeasts: 3 . Methods and strategies used for sequence analysis and annotation. FEBS Lett 2000, 487:17-30.

31. Rouhier N, Jacquot JP: Plant peroxiredoxins: alternative hydroperoxide scavenging enzymes. Photosynth Res 2002, 74:259-268.

32. Jeong JS, Kwon SJ, Kang SW, Rhee SG, Kim K: Purification and characterization of a second type thioredoxin peroxidase (type II TPx) from Saccharomyces cerevisiae. Biochem 1999, 38:776-783

33. Christman MF, Morgan RW, Jacobson FS, Ames BN: Positive control of a regulon for defenses against oxidative stress and some heat-shock proteins in Salmonella typhimurium. Cell 1985, 41:753-762.

34. Armstrong-Buisseret L, Cole MB, Stewart GS: A homologue to the Escherichia coli alkyl hydroperoxide reductase AhpC is induced by osmotic upshock in Staphylococcus aureus. Microbiol I995, I4 I: 1655-I66I.

35. Leblanc L, Leboeuf C, Leroi F, Hartke A, Auffray Y: Comparison between $\mathrm{NaCl}$ tolerance response and acclimation to cold temperature in Shewanella putrefaciens. Curr Microbiol 2003, 46:157-162.

36. Chauhan R, Mande SC: Characterization of the Mycobacterium tuberculosis H37Rv alkyl hydroperoxidase AhpC points to the importance of ionic interactions in oligomerization and activity. Biochem I 200I, 354:209-2I5.

37. Rhee HJ, Kim GY, Huh JW, Kim SW, Na DS: Annexin I is a stress protein induced by heat, oxidative stress and a sulfhydrylreactive agent. Eur J Biochem 2000, 267:3220-3225.

38. Irani K, Xia Y, Zweier JL, Sollott SJ, Der CJ, Fearon ER, Sundaresan M, Finkel T, Goldschmidt-Clermont PJ: Mitogenic signaling mediated by oxidants in Ras-transformed fibroblasts. Science 1997, 275: 1649-1652. 
39. Yuan L, Hillman JD, Progulske-Fox A: Microarray analysis of quorum-sensing-regulated genes in Porphyromonas gingivalis. Infect Immun 2005, 73:4I 46-4I54.

40. Tanaka Y, Hibino T, Hagashi Y, Tanaka A, Kishitani S, Takabe T, Yokota $S$, Takabe T: Salt tolerance of transgenic rice overexpressing yeast mitochondrial Mn-SOD in chloroplast. Plant Sci 1999, I 48: $131-138$

4I. Roxas VP, Lodhi SA, Garrett DK, Mahan JR, Allen RD: Stress tolerance in transgenic tobacco seedlings that overexpress glutathione S-transferase/glutathione peroxidase. Plant Cell Reports 2000, 42: 1229-1234.

42. Schmitt ME, Brown TA, Trumpower BL: A rapid and simple method for preparation of RNA from Saccharomyces cerevisiae. Nucleic Acids Res 1990, 18:3091-3092.

43. Del Aguila EM, Dutra MB, Silva JT, Paschoalin VM: Comparing protocols for preparation of DNA-free total yeast RNA suitable for RT-PCR. BMC Mol Biol 2005, 6:9-15.

44. Hoffman CS, Winston F: A ten-minute DNA preparation from yeast efficiently releases autonomous plasmids for transformation of Escherichia coli. Gene 1987, 57:267-72.

45. Tarutina MG, Tolstorukov II: Development of a method for the vector transformation of the methylotrophic yeast Pichia methanolica. Russian J of Genetics 1994, 30:689-695.

46. Chattopadhyay MK, Tabor CW, Tabor H: Polyamine deficiency leads to accumulation of reactive oxygen species in a spe2 mutant of Saccharmyces cerevisiae. Yeast 2006, 23:75I-76I.

Publish with Bio Med Central and every scientist can read your work free of charge

"BioMed Central will be the most significant development for disseminating the results of biomedical research in our lifetime. "

Sir Paul Nurse, Cancer Research UK

Your research papers will be:

- available free of charge to the entire biomedical community

- peer reviewed and published immediately upon acceptance

- cited in PubMed and archived on PubMed Central

- yours - you keep the copyright

Submit your manuscript here:

http://www.biomedcentral.com/info/publishing_adv.asp
BiolMedcentral 